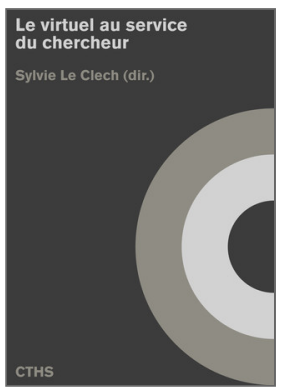

Sylvie Le Clech (dir.)

Le virtuel au service du chercheur

Éditions du Comité des travaux historiques et scientifiques

\title{
La genèse des discours journalistiques sur les musées et les expositions virtuels
}

\section{Annick Batard}

DOI : $10.4000 /$ books.cths. 15298

Éditeur : Éditions du Comité des travaux historiques et scientifiques Lieu d'édition : Éditions du Comité des travaux historiques et scientifiques Année d'édition : 2021

Date de mise en ligne : 21 juin 2021

Collection : Actes des congrès nationaux des sociétés historiques et scientifiques EAN électronique : 9782735509300

\section{Qb boooks}

http://books.openedition.org

\section{Référence électronique}

BATARD, Annick. La genèse des discours journalistiques sur les musées et les expositions virtuels In : Le virtuel au service du chercheur [en ligne]. Paris : Éditions du Comité des travaux historiques et scientifiques, 2021 (généré le 24 juin 2021). Disponible sur Internet : <http://books.openedition.org/ cths/15298>. ISBN : 9782735509300 . DOI : https://doi.org/10.4000/books.cths. 15298. 


\title{
La genèse des discours journalistiques sur les musées et les expositions virtuels
}

\author{
Annick Batard
}

1 Les musées - établissements fondés sur la conservation, l'exposition, que Jean Davallon considère comme un média ${ }^{1}$, et la valorisation des œuvres d'art - sont généralement ancrés dans le réel. La problématique s'intéressera aux discours journalistiques sur les musées qui s'emparent de ce thème du musée virtuel, au moment du passage de la monstration des œuvres physiques à la numérisation et à la disposition de celles-ci sur des disques multimédias, dans le milieu des années 1990. En effet, à partir d'éléments de notre thèse ${ }^{2}$ portant sur la critique journalistique - éphémère, mais qui a légitimé les CD-ROM et les jeux video ${ }^{3}$ - nous questionnerons cette apparition des termes « musées virtuels » ou encore « expositions virtuelles », renvoyant à des supports et des contenus multimédias.

2 Aujourd'hui, les musées ont une politique dynamique de présence sur le Net. Nous interrogerons donc la genèse des discours concernant les aspects multimédias des musées auprès du grand public. En effet, la critique des CD-ROM et des jeux vidéo s'est largement intéressée à ceux consacrés aux musées, notamment le Louvre ou Orsay. La presse généraliste rendait alors compte des collections des musées et des expositions, ainsi que de leur dimension multimédia, et exerçait ainsi une certaine pédagogie envers les lecteurs peu familiers de ces notions à l'époque. Nous verrons qu'il existait une certaine interaction entre le réel - un musée, une exposition - et le virtuel - un CDROM ou l'annonce de la création du site d'un musée.

\section{Éléments contextuels}

Questionner la genèse des discours journalistiques sur le multimédia relatifs aux musées et aux expositions suppose quelques rappels de contexte. En France, le milieu des années 1990 voit l'apparition des éléments relatifs au multimédia et à Internet. Sont 
alors évoqués les multimédias en ligne ou hors ligne, pour distinguer ce qui est connecté de ce qui est édité sur un support autre que le papier. Nos interrogations sur la légitimation des CD-ROM par le biais d'une critique journalistique, inspirée pour partie de la critique littéraire ${ }^{4}$, nous ont conduit à constater que le multimédia relatif aux musées et aux expositions compte pour une part importante dans les discours sur ces nouveaux produits culturels multimédias. À l'époque, certains éditeurs de CD-ROM connaissent d'ailleurs un grand succès, dont la presse généraliste contemporaine française se fait le relai. Au milieu des années 1990, les premiers discours journalistiques évoquent la question du matériel nécessaire pour consulter ces nouveaux produits multimédias, indiquent qu'ils restent relativement coûteux mais conseillent pourtant aux lecteurs de s'équiper. N'oublions pas non plus le soutien des pouvoirs publics, très favorables au multimédia à l'époque. Le rapport Théry indique ainsi : "La visite en multimédia ne remplacera pas la visite réelle. Elle la suscitera, l'attrait du virtuel appelant immanquablement le désir du réel ${ }^{5}$."

Les discours journalistiques commencent à parler de manière régulière des CD-ROM et jeux vidéo destinés au grand public à l'hiver 1994, avec une préfiguration dès 1992. Le Nouvel Observateur (devenu L'Obs depuis lors) écrit :

«Pour réaliser son rêve culturel et afficher son goût de la peinture, Bill Gates n'y est pas allé par quatre chemins: pendant une dizaine d'années, il a consciencieusement dévalisé les plus grands musées du monde, achetant pour son propre compte les droits de reproduction de leurs chefs-d'œuvre, par l'intermédiaire de sa société Home System Interactive. Il y a quelque temps, il a cédé Home System Interactive à Microsoft, qui s'est aussitôt lancé dans l'édition de CD-ROM. Le musée chez soi était né. La vogue outre-Atlantique, des home theater TV, ces immenses écrans qui donnent toute leur valeur aux grands spectacles ou aux belles choses, a contribué à la populariser. [...] De son côté, la Réunion des musées nationaux (RMN) a fait éditer, à l'occasion de l'exposition consacrée par le musée d'Orsay aux origines de l'impressionnisme, un disque optique assez détaillé ${ }^{6}$. "

5 Cet extrait indique bien l'intérêt pour les contenus culturels et leur diffusion sur de nouveaux supports, et précise que de nouveaux acteurs venus du monde de l'informatique ou des télécommunications n'hésitent pas à largement numériser et utiliser les contenus disponibles dans les musées pour proposer leurs offres de titres multimédias. Un article du Monde souligne aussi qu'un accord a été pris entre la RMN et une filiale de France Télécom, pour la «valorisation du patrimoine européen par les médias électroniques" ", afin d'essayer de lutter contre l'hégémonie des Américains.

6 L'effervescence en matière de CD-ROM et de jeux vidéo se poursuivra du milieu des années 1990 jusqu'au tournant du XxI ${ }^{\mathrm{e}}$ siècle. La production de titres sera très diverse et hétérogène, proposant aussi bien des titres savants - visites de musées, encyclopédies... - que plus populaires, allant de contenus culturels ou para-culturels, en passant par des titres ludo-éducatifs, ainsi qu'un certain nombre de jeux vidéo, plus ou moins violents. Françoise Séguy souligne d'ailleurs «la faiblesse du maillon conceptuel $^{8} »$ de ces produits multimédias.

\section{La contribution du musée du Louvre à la réussite du multimédia}

7 Un aspect du multimédia, qui doit beaucoup aux savoirs et pratiques muséographiques, est popularisé par la presse généraliste. Il réside dans la question de la visite virtuelle 
d'un musée ou d'une exposition. La technologie se met ainsi au service des musées et permet de mieux faire connaitre leurs collections et expositions. Le CD-ROM devient alors un élément ajouté à la stratégie commerciale, au même titre que le catalogue édité sur papier à cette occasion.

8 Le CD-ROM du Louvre, édité par Montparnasse Multimédia, filiale des Éditions Montparnasse, spécialiste de vidéos que l'on pourrait qualifier de classiques - comme celles de Marcel Carné, Jacques Prévert, Jean Renoir, Louis Jouvet ou Jean Gabin -, fait l'objet d'un grand lancement événementiel en novembre 1994, dans le lieu même du musée du Louvre. Cette soirée de relations publiques signe, en quelque sorte, le démarrage des articles de la presse généraliste. La concomitance entre ce grand événement - qui participe ainsi aux stratégies de promotion et de communication que déploient les industriels des CD-ROM - et la publication des premières critiques journalistiques consacrées aux CD-ROM dans la presse généraliste, indique bien que les contenus culturels et notamment muséaux sont susceptibles de provoquer le vif intérêt des médias et du grand public. Bernard Miège et Yves de La Haye ont d'ailleurs montré que les industriels souhaitaient " préparer les marchés et préparer les esprits ${ }^{9}$ » afin de créer la demande d'un produit pour un marché alors inexistant.

Les journalistes soulignent la déambulation virtuelle et se réjouissent du nombre d'œuvres présentées par le musée dans le disque : «Le Louvre à domicile, ou comment errer, de façon simple et instructive, dans les salles de musée, à la découverte de près de deux-cents œuvres (sculptures, objets d'art) ${ }^{10}$.» Télérama écrit: «Dans un disque, par exemple, on visite à l'envi le musée du Louvre (instructif); dans un autre, on se promène à travers les œuvres de Nicolas Poussin (complet et magnifique). On apprend

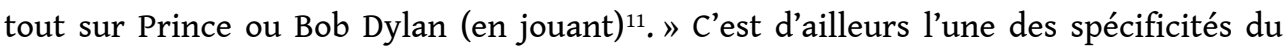
multimédia que d'éditer des œuvres très diverses, allant de produits de qualité à d'autres titres moins exigeants, et de mélanger arguments de légitimité culturelle et plaisir ludique.

Intitulé « Le Louvre chez soi », l'article de La Croix est enthousiaste :

«Le développement des CD-ROM connaît quelques belles réussites. L'une des plus originales concerne l'un des plus célèbres musées du monde : le Louvre. Il aura fallu huit mois de travail à la société Montparnasse Multimédia, en coédition avec la RMN, pour mener à bien ce projet de visite interactive. Le résultat est saisissant ! On peut tout d'abord choisir le sens de son exploration, commencer par le palais, s'engager dans les différentes parties et entrer dans les salles. Il suffit alors de cliquer sur les tableaux proposés - ils sont au nombre de 100 dans ce premier volume - pour les voir apparaître en plein écran. Outre les explications concernant la biographie du peintre, le menu vous propose une loupe pour vous promener comme vous le souhaitez dans les détails de l'œuvre ${ }^{12}$. »

11 Le Figaro célèbre lui aussi le CD-ROM du Louvre dans un article intitulé « La Joconde à domicile ${ }^{13} »$ :

« Fini le temps où je devais empiler sur mon bureau les volumes des encyclopédies pour trouver des informations complètes d'abord sur le Louvre, puis sur les tableaux du musée. Avec le nouveau CD-ROM sur Le Louvre : peintures et palais [...], je peux aisément obtenir la majeure partie des informations que je désire. Pas toutes, malheureusement, car ce CD-ROM ne concerne qu'un choix des plus célèbres peintures. Parmi celles-ci, la fameuse Joconde ${ }^{14}$. Là, on découvre une des potentialités de ce nouveau média : j'ai pour moi tout seul la Joconde à domicile. Sans la cohue des Japonais, ni la vitre blindée qui la protège. En plus, je peux l'observer à satiété sans être dérangé et suivre les explications sur son élaboration ${ }^{15}$." 
Cette idée d'avoir le musée chez soi n'est pas nouvelle. En 1913, l'inspecteur général des Beaux-Arts Armand Dayot s'extasiait déjà des possibilités offertes par les innovations techniques et notamment celle de la photographie en couleur, permettant un meilleur regard sur les œuvres des musées :

«Posséder les musées chez soi, à portée de la main à toute heure, à tout instant, quelle heureuse fortune! Le musée chez soi, c'est-à-dire la possibilité d'évoquer la fidèle image de la toile qu'on aime, de faire revivre et prolonger à son gré l'émotion ressentie devant les œuvres admirées jadis et qu'on ne reverra peut-être plus! Quel précieux privilège de pouvoir contempler ainsi tous les tableaux célèbres, épars dans les musées les plus lointains, à Madrid, à Florence, à Londres.

Et quel concours miraculeux nous fournit la science moderne !

La photographie noire, sans lumière, uniforme de tons, a désormais vécu pour faire place à la photographie des couleurs. Ah l'admirable découverte, en vérité ! Grâce à elle, la peinture nous est restituée tout entière ; c'est le tableau qui revit sous nos yeux. [...] Cela n'est-il pas merveilleux, et n'est-il pas juste de dire que, possédant un tel ouvrage, on possède réellement le musée chez soi ${ }^{16}$ ? "

Déjà à l'époque, les commentateurs s'enthousiasment pour les techniques nouvelles, admirent le côté spectaculaire du recueil et apprécient grandement le fait de pouvoir en bénéficier à domicile. Propos d'une étonnante modernité.

Revenons au Louvre et à la sortie du CD-ROM qui lui est consacré dans le milieu des années 1990. L'article de Libération, publié environ quatre mois après la soirée de lancement, aborde le succès commercial du CD-ROM : environ 500000 exemplaires ont été vendus en quelques années. Le sous-titre de l'article précise : «Fait exceptionnel, un CD-ROM culturel rivalise avec les meilleurs jeux. Les innovations imaginées par ses

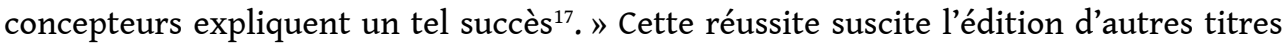
concurrents, remportant plus ou moins de succès. Les années passent et l'intérêt ne se dément pas. Lorsque la technologie se modifie et devient celle du DVD-ROM, les journaux tels que Le Figaro, Le Monde ou Le Parisien, en font également largement écho.

Les discours journalistiques de l'époque sont à la fois assez enthousiastes pour célébrer ces nouveaux supports de contenus culturels et muséaux que sont les CD-ROM, tout en notant parfois certains défauts, comme cela incombe à l'exercice critique ${ }^{18}$.

\section{Le musée d'Orsay se met aussi au multimédia}

Le Louvre n'est pas le seul musée à présenter certains de ses contenus sur des CD-ROM qui suscitent l'intérêt des journalistes. Le musée d'Orsay publie lui aussi un CD-ROM intitulé Musée d'Orsay: visite virtuelle, avec l'éditeur Montparnasse Multimédia et la RMN. Gérard Pangon de Télérama indique à ce propos : « Nous voilà maintenant conviés à une visite virtuelle du musée d'Orsay, dont le petit monde multimédiatique a les oreilles rebattues depuis quelques mois: "Les progrès techniques sont énormes, vous allez voir ce que vous allez voir." 19 "

Un grand article du Monde, intitulé « Orsay : les trésors d'un musée virtuel ${ }^{20}$ », souligne certains défauts du CD-ROM - «la sophistication [...] toute relative, la définition de l'image [...] pas parfaite, les tableaux [...] peu visibles ${ }^{21} »-$ mais insiste globalement sur l'aspect virtuel de la visite. Les journalistes écrivent :

"Calé dans son fauteuil, le nouvel amateur d'œuvres d'art parcourt les musées sans bouger de chez lui. Un écran interactif et une souris lui suffisent. Le voyage culturel immobile commencé avec Le Louvre se poursuit avec Orsay. Deux ans séparent les 
deux visites : en réalité un monde. Non seulement le curieux de 1996 peut passer à Orsay de salle en salle - ce qui lui était interdit en 1993-, mais il a même la possibilité de remonter le temps et d'arpenter les anciens quais de la gare. Les possibilités qu'offre à l'utilisateur le CD-ROM consacré au musée d'Orsay sont infiniment plus riches que celles données par le CD-ROM du Louvre. Les œuvres qu'il présente sont plus nombreuses (100 tableaux pour le précédent, 200 toiles et sculptures pour le nouveau). Pas d'images virtuelles dans le premier. Elles sont esquissées dans le second. Autre nouveauté prometteuse incluse dans Orsay, le "guide intelligent", qui propose des visites de musée "à la carte", en fonction des goûts de l'utilisateur [...]. Alors que Le Louvre se borne pratiquement à un simple catalogue, à Orsay, les œuvres disséquées sont accompagnées d'une centaine de biographies d'artistes et surtout de récits transversaux, tranches d'histoire de l'art ou aperçu sur quelques mouvements artistiques. [...] Pourtant, la sophistication d'Orsay est encore toute relative. La définition des tableaux n'est pas parfaite, les tableaux "accrochés" dans les salles que le visiteur peut parcourir sont peu visibles, l'effet loupe fait apparaître une vilaine trame.»

Le Figaro choisit de parler du CD-ROM du musée d'Orsay en présentant le portrait d'un des pionniers du multimédia, Pierre Raiman ${ }^{22}$, qui a participé à la création de Montparnasse Multimédia à l'été 1992, société éditrice de ces deux CD-ROM muséaux.

\section{Une catégorie à part : le jeu culturel}

19 Une catégorie spécifiquement française, liée à l'univers muséal et culturel, a connu durant un certain nombre d'années un vif succès, critique et commercial. Le CD-ROM Versailles 1685: complot à la cour du Roi-Soleil - édité chez Cryo Interactive, avec le concours de la RMN et de la conservation du château de Versailles, ainsi que des éditions Textuel et de Canal+- fait l'objet d'une belle critique dans le supplément multimédia alors mis en place par le quotidien Libération, qui souligne que le joueur est projeté dans l'univers du Roi-Soleil dans «un château en image de synthèse à la précision historique étonnante ${ }^{23} »$. Laure Pelé ajoute dans Le Parisien :

«Ce qui rend ce CD-ROM intéressant, c'est la minutieuse reconstitution en 3D du château, tel qu'il était en 1685 . C'est-à-dire complètement différent de celui d'aujourd'hui. Ainsi, vous découvrirez des lieux et objets disparus ou interdits à la visite comme le magnifique escalier des Ambassadeurs, le labyrinthe dans le jardin ou le mobilier d'argent ${ }^{24}$. "

20 En effet, dès cette époque, que ce soit sous la forme d'un jeu ou d'un CD-ROM culturel, les titres des articles évoquent les possibilités offertes par le multimédia afin de reconstituer et de modéliser des éléments historiques disparus, et ce de manière ludique.

Un autre jeu culturel intitulé Le Louvre: l'ultime malédiction, est produit en 2000 par Index+, la RMN et France Télécom Multimédia. À son sujet, la critique précise que le scénario est « ingénieux » et que le joueur est en « totale immersion dans des décors en 3D précalculées qui permettent une navigation à 360 degrés ». En effet, "quarante pièces oubliées du Louvre ont donc été modélisées. Seules deux salles n'ont pas connu les affres du temps. Un important travail de reconstitution a été effectué pour rendre ce jeu historiquement plausible. Des archéologues ont communiqué les plans au sol. [...] Après avoir découvert le Louvre historique grâce à ce jeu, c'est un autre regard que l'on porte sur le musée ${ }^{25} »$. Les interactions entre culture et divertissement sont ainsi de nouveau évoquées. 
La catégorie des jeux culturels disparait pourtant rapidement au profit des jeux vidéo. Ces derniers continuent à être critiqués par la presse écrite, même si ce n'est plus sous la même forme organisée et régulière qu'auparavant. Cet examen permet d'apprécier les contenus et de souligner les innovations technologiques, mais aussi de se comparer aux musées, et en l'occurrence au Louvre. Le journaliste Benjamin Guez du Point note la faiblesse du scénario du jeu The Crystal Key, édité par Alsyd, mais souligne en revanche la beauté de ses décors:

«La technologie QuickTime utilisée par les créateurs de The Crystal Key est pour beaucoup dans les images exceptionnelles. Celle-ci permet une liberté de mouvement complète et un déplacement à 360 degrés. On se croirait en pleine visite virtuelle du Louvre, qui utilise pour son site le même procédée ${ }^{26}$. »

L'intertitre reprend d'ailleurs la comparaison avec la visite virtuelle du Louvre pour bien inscrire le jeu dans l'univers culturel.

\section{Des expositions réelles et/ou virtuelles}

4 L'engouement pour les expositions, notamment celle consacrée au peintre Cézanne par le Grand Palais en 1996, conduit à l'édition de trois CD-ROM aux succès inégaux. Le CDROM Moi, Paul Cézanne, réalisé avec le concours de la RMN et de Télérama est une réussite (25000 exemplaires vendus en trois mois). À ce propos, Sylvie Metzelard du Parisien indique: «Si ces promenades virtuelles à travers une exposition plaisent de plus en plus, elles ne dament pas pour autant le pion aux visites sur place ${ }^{27}$.» Daniel Ichbiah, spécialiste des nouvelles technologies, partage le même avis : «Au bout du compte, et contrairement à ce que pourraient penser les technophobes, un CD-ROM d'une telle qualité [Le Louvre] donne envie de foncer voir les originaux ${ }^{28}$.» La complémentarité entre œuvres exposées et représentées fonctionne.

Un article du Monde précise combien le multimédia constitue un outil au service de l'histoire et des institutions culturelles et muséales: «Enquête: la réalité virtuelle explore les trésors culturels du passé.» Le chapeau explique: «Cavernes préhistoriques interdites au public, cités rêvées, basiliques disparues : des chercheurs mobilisent érudition et technologies pour reconstituer les pans engloutis du patrimoine artistique: grottes de Lascaux et Cosquer, tombeau de Néfertari, "ville idéale" de Giotto, ou encore Montpellier à travers les siècles, main dans la main avec un François Rabelais virtuel ${ }^{29}$.»

Une profusion de titres de CD-ROM culturels est donc critiquée par la presse généraliste française et souligne l'intérêt que peut représenter le multimédia à l'époque. Ainsi, à propos d'un CD-ROM Gauguin écrivain en 2003 et de l'exposition qu'il accompagne, Dorothé Thorel écrit-elle dans Le Point :

«La consultation des manuscrits étant interdite au grand public du fait de leur fragilité, la Réunion des musées nationaux et le musée d'Orsay proposent désormais un CD-ROM contenant le facsimilé numérique de trois manuscrits presque inconnus: "Ancien culte mahorie", "Noa Noa" et "Diverses choses" [...]. L'écriture lisible et simple du peintre dans les deux premiers textes contribue à la grande sobriété du CD-ROM, au sein duquel la navigation est fluide. [...] Un magnifique plongeon dans cette "Navenave fenua. Noanoa": terre délicieuse, terre odorante ${ }^{30}$.»

La fragilité des matériaux réels donne alors tout son sens à l'édition d'un CD-ROM multimédia, qui peut profiter au plus grand nombre. 
Un thème, toujours fort prisé des Français, connaît également un grand succès en matière de multimédia : celui de l'égyptologie ${ }^{31}$. Trop de CD-ROM sont pourtant édités en 2001 : «Sacrés pharaons! [...] Huit titres jouent des coudes pour vous apprendre à distinguer Néfertiti de Nofertari, savoir à quoi ressemble le temps d'Horus ou connaître la différence entre le Moyen et le Nouvel Empire ${ }^{32}$. » Les journalistes précisent toutefois que les différents titres ne sont pas équivalents en matière de connaissances et de sérieux scientifique. À propos du CD-ROM supervisé par Christiane DesrochesNoblecourt - d'ailleurs jugé «savant » par les journalistes du Point évoqués ci-devant -, L'Humanité insiste, par le biais d'une interview avec la célèbre égyptologue, sur certaines possibilités offertes par le multimédia, notamment en ce qui concerne la reconstitution d'aspects historiques. Christiane Desroches-Noblecourt y affirme sa volonté de diffusion de la science :

«Avec ce simple outil - un de plus parmi d'autres - on peut faire des images même virtuelles très proches de la réalité. Il a été possible d'animer les bas-reliefs des tombes de façon à rendre compte de la vie des courtisans du temple; grâce aux monuments de Toutankhamon, aux objets du trésor et aux photos, reprises en couleurs, on peut évoquer toute une histoire [...] Tout cela a été reconstitué avec des éléments réels, des objets, des paysages et des monuments d'Égypte. [...] Souci iconographique du moindre détail, le produit est soigné ${ }^{33}$. »

\section{De nouveaux termes apparaissent}

Nous avons eu l'occasion d'indiquer dans notre thèse que de nouvelles normes multimédias s'organisent autour de quatre grands concepts: le rapport homme/ machine, le déplacement, la représentation et la mise en relation ${ }^{34}$. Ces nouveaux critères et ces nouveaux termes sont peu à peu examinés et expliqués par les journalistes. Ainsi, l'interface, la navigation, l'interactivité, le clic, le zoom et autres éléments deviennent peu à peu familiers au grand public. Citons un exemple :

«Les interfaces, cet ensemble de flèches et boutons qui permettent d'avancer dans la découverte du CD-ROM, sont plus fonctionnelles et plus discrètes. Le temps est révolu de ces toiles de maîtres "mangées" par une prolifération d'instruments de navigation $^{35}$.»

« Nettement plus spectaculaire, la visite virtuelle de l'Égypte au Louvre de la société Emme/Acta. Nous sommes ici plongés dans un univers en 3D par le biais d'une caméra omni-dimensionnelle. Les objets sont cliquables ${ }^{36}$, les couloirs défilent dans une fluidité parfaite ${ }^{37}$.»

$\mathrm{Au}$ fil des années, une préparation et une pédagogie des nouveaux mots et usages du numérique sont mises en place par des journaux généralistes - visite virtuelle, musée ou exposition virtuels et même l'oxymore "réalité virtuelle», déplacement en 3D, zoom, modélisation, navigation, interactivité... - contribuant à enrichir le vocabulaire et la patrimonialisation des œuvres d'art et du passé. La muséographie d'aujourd'hui s'est emparée de ces nouveaux outils et dispositifs pour le plus grand plaisir des visiteurs. 


\section{Prolongement sur Internet et progressive disparition des CD-ROM} celui du prolongement sur Internet. Ce point révèle les imbrications qui existent à l'époque entre le CD-ROM et le réseau Internet. Ces liens contribuent en quelque sorte à effectuer une transition entre la critique de CD-ROM et la critique de sites Internet, et vont de pair avec les discours abondants sur le réseau. Donnons un exemple avec le CDROM déjà présenté sur l'exposition Paul Cézanne :

«On peut également se connecter sur l'expo à succès de l'année avec Internet. Et, la semaine dernière, on recensait déjà 77000 appels. Internet où l'on retrouve, entre autres, les œuvres essentielles présentées au Grand Palais, le nombre de visiteurs, les adresses et téléphones utiles, un forum où poser des questions ${ }^{38}$... »

Un article de Libération au titre humoristique («le CD-ROM perd connaissance») souligne, dès 2001, la progressive disparition des CD-ROM. Le même article invoque une interprétation donnée par le cabinet GFK : «L'essor de l'Internet, cette bibliothèque publique universelle [...] transformerait les $\mathrm{CD}$ en objets inutiles ». Il explique aussi que le succès du CD-ROM Le Louvre, vendu à plusieurs centaines de milliers d'exemplaires, «a totalement perturbé le marché en faisant croire que ce segment existait. En fait, il n'y a pas de réelle valeur d'usage du CD-ROM culturel ${ }^{39}$. " Le CD-ROM « est comme les livres d'art, culturellement nécessaire, même s'il n'est pas rentable économiquement ${ }^{40}$.» Propos précurseurs! Progressivement d'ailleurs, la rubrique multimédia de certains journaux - Libération, Le Monde, Télérama - évoquera des sites Internet de musées ou d'initiatives culturelles et patrimoniales, et peu à peu ne parlera plus des CD-ROM.

Aujourd'hui, l'importante production de CD-ROM comportant des contenus culturels a disparu, emportant avec elle sa critique journalistique. En revanche, les dispositifs numériques se sont beaucoup développés. De nombreux musées proposent des écrans permettant de voir les reconstitutions en 3D de certains aspects architecturaux et objets disparus, et ce à plusieurs époques de l'histoire. Les mots et ce qu'ils recouvrent en matière de possibilités liées au numérique comme " clic ", « zoom », " navigation ", « modélisation ", "visite virtuelle » ou même "réalité virtuelle », sont entrés dans le langage courant. Les CD-ROM consacrés aux musées et aux expositions, et leur critique journalistique, ont donc bien rempli leur rôle de pédagogie et d'accompagnement des termes et des pratiques durant une dizaine d'années. La muséographie a ajouté à sa panoplie d'outils différents dispositifs numériques, multimédias et interactifs, qui rivalisent de contenus aux savoirs scientifiques, avec les possibilités offertes par les techniques et les savoir-faire en matière de mises en scène muséographiques. D'ailleurs, un article de Marc Terrisse ${ }^{41}$, qui interroge les évolutions et possibilités de développement des musées et visites virtuelles ${ }^{42}$, en détaillant notamment le Google Art Project, précise que les innovations techniques n'invalident pas les usages identifiés dès le début - complémentarité du musée réel et de la visite virtuelle, faculté d'examen en cas d'impossibilité d'accès aux lieux ou aux matériaux trop fragiles...-, comme les écrits journalistiques de notre corpus l'ont également souligné à leur époque. 


\section{BIBLIOGRAPHIE}

BATARD Annick, « La critique des expositions et des musées en France », Médiations et Informations, $\mathrm{n}^{\text {os }} 42-43$, Mitropoulou Eleni et Novello Paglianti Nanta (dir.), Exposition et communication, 2018, p. 55-68.

BATARD Annick, « La critique journalistique des CD-ROM “culturels" : entre promotion commerciale et invention d'un genre ", thèse de doctorat en sciences de l'information et de la communication, Paris, université Paris XIII - Paris-Nord, 2003 (2 vol.).

BRUZEK Olivier et AVRAND-MARGOT Sylvia, « Les CD-ROM d'égyptologie », Le Point, 29 juin 2001.

CLOUZOTTE Guy, «Visite au musée virtuel », Le Nouvel Observateur-PC Direct-PC Expert, $\mathrm{n}^{\circ} 1568$, 24 novembre 1994.

ColonNA Jean-Jacques, « Le multimédia fou d'Égypte », Le Figaro, $1^{\text {er }}$ décembre 1999.

COLONNA D’ISTRIA Michel, « Les vertus des musées virtuels », Le Monde, 22 février 1994.

DANIEL Nicolas, « Avec Toutankhamon, Christiane Desroches-Noblecourt présente l'Égypte sur CDROM », entretien, L'Humanité, 25 novembre 1997.

DAVALLON Jean, L'exposition à l'œuvre : stratégie de communication et médiation symbolique, Paris, L'Harmattan, 1999.

DAYOT Armand, inspecteur général des Beaux-Arts (dir.), Les grands musées du monde illustrés en couleurs : le musée du Louvre, préf. Armand Dayot, Paris, éditions Pierre Laffite, 1913 (2 vol.).

EUDES Yves et ROUX Emmanuel de, « Orsay : les trésors d'un musée virtuel », Le Monde, 18 février 1996.

EUDES Yves et Roux Emmanuel de, « Une visite dans un fauteuil », Le Monde, 19 février1996.

GEOFFREY Flore, « La souris et la Joconde », Télérama, nº 2367, 24 mai 1995.

GUEZ Benjamin, «Qu'importe le flacon », Le Point, $\mathrm{n}^{\circ}$ 1500, 15 juin 2001.

GUILMONT Marie-Véronique, « Le Louvre, l'ultime malédiction!", Le Point : édition grandes écoles et universités, $\mathrm{n}^{\circ} 1442,5$ mai 2000.

ICHBIAH Daniel, « Évasion et émotion à domicile », Le Nouvel Observateur-PC Direct-PC Expert, 24 novembre 1994.

LA HAYE Yves de, Dissonances : critique de la communication, introd. Bernard Miège, Grenoble, La Pensée Sauvage, 1984.

LAGRANGE Roger-Pierre, « La guerre des Cézanne », Libération, 22 septembre 1995.

LAGRANGE Roger-Pierre, « Le Louvre, triomphe d'un musée imaginaire », Libération, 10 mars 1995.

LATRIVE Florent et PEYRET Emmanuèle, « Versailles story », Libération, 25 octobre 1996.

LE LEURCH Vincent, « Faut-il céder aux CD-ROM ?», Télérama, n 2363, 26 avril 1995.

LE TELLIER Hervé, Joconde sur votre indulgence, Bordeaux, Le Castor astral, 2002.

LEMIRE Laurent, « Le Louvre chez soi », La Croix, 28 décembre 1994.

MALET Caroline de, « CD-ROM Louvre : le musée virtuel », Le Figaro, $1^{\mathrm{er}}$ avril 1996. 
MALPHETTES Stéphanie, « Les nouveaux musées imaginaires », Le Monde TRM, 21-22 juillet 1994.

MÉEuS Carl, « Tous les chemins mènent au CD-ROM », Le Figaro, 28 novembre 1994.

METZELARD Sylvie, « Le CD-ROM Cézanne s'arrache », Le Parisien, 10 octobre 1995.

PANGON Gérard, « Orsay, presque comme si vous y étiez », Télérama, n 2411, 27 mars 1996.

PELÉ Laure, « Le château de Versailles sur CD-ROM ! », Le Parisien, 28 octobre 1996.

RIVOIRE Annick, « Le CD-ROM perd connaissance », Libération, 4 juin 2001.

SÉGUY Françoise, Les produits interactifs et multimédias, Grenoble, Presses universitaires de Grenoble, 1999.

TERRISSE Marc, « Musées et visites virtuelles : évolutions et possibilités de développement », Muséologies, vol. VI, nº 2, p. 15-32. [URL : https://doi.org/10.7202/1018927ar]

THÉRY Gérard, Les autoroutes de l'information : rapport au Premier ministre, Paris, La Documentation française, 1994.

THOREL Dorothé, « CD-ROM Gauguin écrivain », Le Point, 17 octobre 2003.

WAINTROP Édouard, « Il a mis le Louvre dans votre ordinateur », Libération, 28 décembre 1995.

\section{NOTES}

1. J. Davallon, L'exposition à l'œuvre [...].

2. A. Batard, « La critique journalistique des CD-ROM “culturels" [...]».

3. Le CD-ROM est un disque optique contenant des textes, des images et des sons. Il s'agit d'un support matériel, avec des contenus ayant une dimension culturelle ou paraculturelle. Les jeux vidéo sont aussi des contenus, mais ils deviennent une catégorie à part entière, face aux CD-ROM qui disparaissent progressivement.

4. A. Batard, « La critique journalistique des CD-ROM “culturels" [...] ».

5. G. Théry, Les autoroutes de l'information [...], p. 14.

6. G. Clouzotte, «Visite au musée virtuel ».

7. M. Colonna d'Istria, « Les vertus des musées virtuels ».

8. F. Séguy, Les produits interactifs [...], p. 4.

9. Y. de La Haye, Dissonances [...], p. 128-129.

10. F. Geoffrey, « La souris et la Joconde ».

11. V. Le Leurch, « Faut-il céder aux CD-ROM?».

12. L. Lemire, « Le Louvre chez soi ».

13. La presse généraliste choisit souvent d'illustrer ses propos par des œuvres très connues.

14. À ce propos, citons "Le point de vue du manuel Microsoft » de l'oulipien Hervé Le Tellier qui invente de savoureux jeux littéraires autour de Mona Lisa : «Introduire le tableau dans le cadre. L'icône de l'application Joconda s'affiche sur le bureau [...] », voir H. Le Tellier, Joconde [...], p. 103.

15. C. Méeus, « Tous les chemins mènent au CD-ROM ». 
16. A. Dayot, Les grands musées du monde [...], préf., p. 6-7.

17. R.-P. Lagrange, « Le Louvre, triomphe d'un musée imaginaire ».

18. Nous avons également eu l'occasion d'interroger la critique des expositions et des musées. Voir A. Batard, « La critique des expositions et des musées en France [...] ».

19. G. Pangon, « Orsay, presque comme si vous y étiez ».

20. Y. Eudes et E. de Roux, "Orsay: les trésors d'un musée virtuel ». Id., "Une visite dans un fauteuil ».

21. Ibid.

22. C. de Malet, «CD-ROM Louvre : le musée virtuel ». Pierre Raiman avait déjà fait l'objet d'un grand portrait publié par Libération le 28 décembre 1995 : É. Waintrop, « Il a mis le Louvre dans votre ordinateur $»$.

23. F. Latrive et E. Peyret, « Versailles story ».

24. L. Pelé, « Le château de Versailles sur CD-ROM !".

25. M.-V. Guilmont, « Le Louvre, l'ultime malédiction! ». L'article précise aussi qu'un site dédié permet de présenter le musée et donne accès aux solutions du jeu.

26. B. Guez, «Qu'importe le flacon ».

27. S. Metzelard, « Le CD-ROM Cézanne s'arrache ».

28. D. Ichbiah, « évasion et émotion à domicile ».

29. S. Malphettes, « Les nouveaux musées imaginaires ».

30. D. Thorel, « CD-ROM Gauguin écrivain ».

31. L'exposition "Toutankhamon: le trésor du Pharaon" tenue du 23 mars au 22 septembre 2019 à la Grande Halle de la Villette en est aussi une preuve.

32. O. Bruzek et S. Avrand-Margot, « Les CD-ROM d'égyptologie ».

33. Entretien réalisé par N. Daniel, «Avec Toutankhamon, Christiane DesrochesNoblecourt présente l'Égypte sur CD-ROM ».

34. A. Batard, « La critique journalistique des CD-ROM “culturels” [...] », t. I, p. 347.

35. R.-P. Lagrange, « La guerre des Cézanne ».

36. Notons l'utilisation journalistique du verbe « cliquer » sous la forme d'un adjectif.

37. J.-J. Colonna, « Le multimédia fou d'Égypte ».

38. S. Metzelard, « Le CD-ROM Cézanne s'arrache ».

39. A. Rivoire, « Le CD-ROM perd connaissance ».

40. Ibid.

41. M. Terrisse, « Musées et visites virtuelles [...]».

42. Le confinement participe également au développement des visites virtuelles des musées et des expositions. Ainsi, notons que la XVI ${ }^{\mathrm{e}}$ Nuit européenne des musées du 14 novembre 2020 "se transpose en ligne » (voir La Gazette Drouot, 13 décembre 2020, p. 169) ou "sera virtuelle», comme l'indique Leila Kaddour au journal télévisé de France 2 (France 2, 13h30, 14 novembre 2020). 


\section{RÉSUMÉS}

La communication interroge la genèse des discours journalistiques sur les musées et les expositions virtuels et montre comment la critique de la presse généraliste contemporaine française exerce une certaine pédagogie vis-à-vis du grand public, en s'extasiant sur les possibilités offertes par ces visites virtuelles à domicile - argument déjà relevé presque cent ans auparavant à propos de la photographie en couleur. Les grands musées, notamment le Louvre et Orsay, accompagnés de la Réunion des musées nationaux (RMN), participent à l'aventure et contribuent au succès des disques multimédias, largement relayés par la presse. Des jeux culturels et la passion du public pour l'égyptologie contribuent aussi à l'enthousiasme pour ces visites virtuelles. Tandis que de nouveaux critères, mots et usages, entrent dans le langage courant, les CD-ROM disparaissent quant à eux progressivement avec leur critique journalistique, au profit des sites Internet des musées et des dispositifs numériques muséographiques.

\section{AUTEUR}

\section{ANNICK BATARD}

Maître de conférences en sciences de l'information et de la communication, Laboratoire des sciences de l'information et de la communication (LabSic, EA 1803), université Sorbonne - ParisNord 